\title{
Research on the two-port network based on the NIF
}

\author{
Zheng Chen-da \\ Operation and maintenance department \\ Maintenance Branch of Fujian Electric Power co., LTD. \\ Fuzhou, China \\ dariotzen@hotmail.com
}

\author{
Lan Yan-ning \\ Operation and maintenance department \\ Maintenance Branch of Fujian Electric Power co., LTD. \\ Fuzhou, China
}

\begin{abstract}
The theory of two-port network is an important aspect both of electrical or electronics, in the past, researches of two-port network are mostly about port characteristics, parametric equation or the equivalent circuit derived from the equation parameters. In this paper,an equivalent topology for the multi-terminal network-NIF is introduced, and then derived the establishment condition of two-port network. Issues such as interconnection of two-port networks has been discussed. It proves that the NIF topology has a strong applicability to the two-port network theory. The conclusion of this paper enriches the theory of two-port networks.
\end{abstract}

Keywords—two-port network; four-terminal network; NIF

\section{INTRODUCTION}

Two-port networks have been widely used in many fields that complex network can be split into a number of simple twoport networks, analysis of the two-ports can also be promoted to the multi-port networks $[1,2]$. Therefore, the two-port network theory has important practical value in subjects such as power system analysis; electronic circuits; measurement technology [3-7], etc. In the past, most of the researches on the two-port network focus on the relationship and characteristics of the ports; Integrate networks by the theory of circuit synthesis, or analyzed and designed electrical circuits according to the two-port theory.

Essentially, a two-port network is a four-terminal network as well. When the certain conditions are met, which is the current flowing from one terminal at any time is equal to the current flowing from the other, that network is called a twoport network. However, such a port condition seems too simple and it causes some inconvenience of researching the two-port basic theory. According to previous studies [8-10], a brand new modeling method named nod-to-nod impedance function (NIF) for multi-terminal networks had been proposed. In this paper, the NIF topology is applied to study of passive and linear timeinvariant two-port network. Then explored the set up conditions of two-port network and the conditions after twoport networks connected. From a new perspective, the two-port network theory has been supplemented.

\section{NIF TOPOLOGY OF THE FOUR-TERMINAL NETWORK}

The port characteristics of multi-network can be accurately reflected by NIF topology. There are some similarities between NIF and the so-called black box method, which is, not specifically exploring the specific structure of the object but focusing on the internal coupling that reflected from the external terminals. In this way, observed parameters by a specific NIF topology would meet the same purpose with the measured parameters of actual object. Different from the traditional way, for a certain two-port network, its NIF topology is unique.

For any four-terminal network, its NIF topology is shown in Fig. 1. Terminals of the four-terminal network ' $\mathrm{N}$ ' are named ' 1 ', ' 1 ', ' 2 ' and ' 2 ', each of them consists of a pair of six branches, 'a', 'b', 'c', 'd', 'e', and 'f' will be connected to each other.

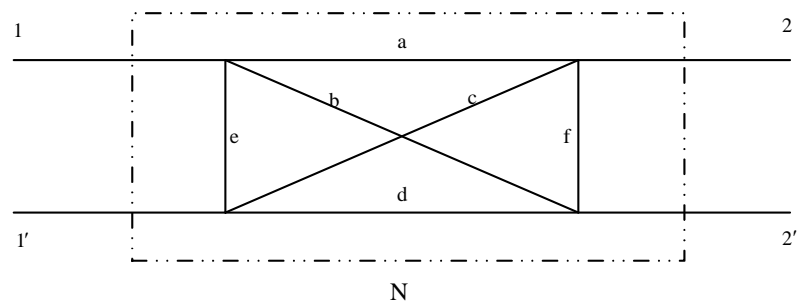

Fig. 1. NIF topology of four terminal network

\section{CONDITIONS FOR THE ESTABLISHMENT OF TWO-PORT NETWORK}

The ' 1 ' and ' 1 ' of the nodes in Fig. 2 are analyzed by KCL, which can be respectively (1) and (2). In the formulas, 'ua', 'ub', 'uc', 'ud' and 'ue' respectively indicate the corresponding branch voltage, and the relevant reference direction of the corresponding branch current is shown in the figure. 'Ya', 'yb', 'yc', 'yd' and 'ye' are the corresponding branch admittances respectively.

$$
\begin{aligned}
& i_{1}=i_{a}+i_{b}+i_{e}=u_{a} y_{a}+u_{b} y_{b}+u_{e} y_{e} \\
& i_{1}=i_{c}+i_{d}+i_{e}=u_{c} y_{c}+u_{d} y_{d}+u_{e} y_{e}
\end{aligned}
$$

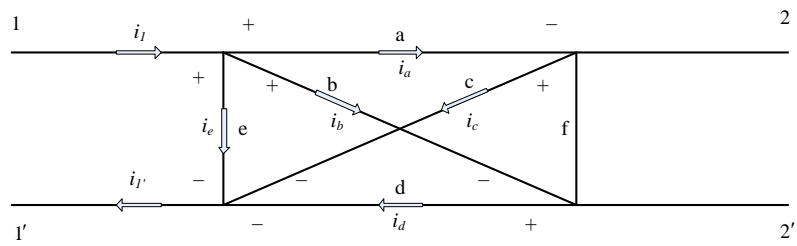

Fig. 2. Diagram of voltage-current in the four-terminal network 
If the four-terminal network ' $\mathrm{N}$ ' satisfies the two-port conditions, current $i_{1}$ and $i_{1}$ ' are equal. Then simultaneous (1) and (2), (3) can be obtained. Similar analysis of the end button ' 2 ' and ' 2 ' can be obtained by the formula (3).

$$
\begin{gathered}
i_{a}+i_{b}=i_{c}+i_{d} \\
u_{a} * y_{a}+u_{b} * y_{b}=u_{c} * y_{c}+u_{d} * y_{d}
\end{gathered}
$$

Transfer the current equation above, it will come to formula (4). Change the reference direction of voltage and current, the formula (4) can be rewritten as (5), as shown in Fig. 3.

The branch ' $\mathrm{e}$ ' and ' $\mathrm{f}$ ' of the two terminals on the end of the connection are defined as the correlation branch, the remaining branches 'a', 'b', 'c' and 'd' as non-correlated branches. Then make a gaussian surface $G$, which fellowship with each non-affiliated branch, and only one intersection. If the sum of current algebra to $\mathrm{G}$ at any time is zero, then the four-terminal network is a two-port network.

$$
\begin{gathered}
i_{a}+i_{b}+\left(-i_{c}\right)+\left(-i_{d}\right)=0 \\
i_{a}+i_{b}+i_{c}+i_{d}=0
\end{gathered}
$$

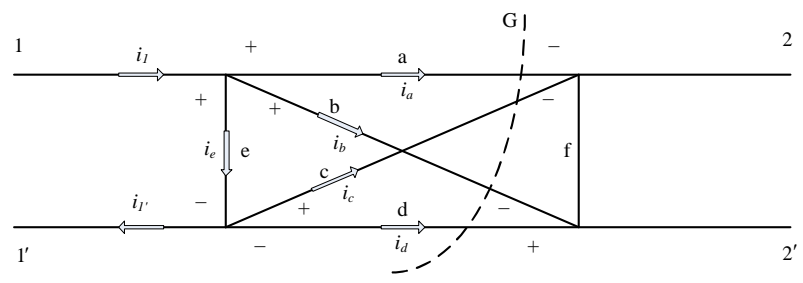

Fig. 3. Diagram of voltage-current in the two-port network

Theoremshe above can be illustrated by a simple example. For the two port network $\mathrm{N}$ in Fig. 4, if there is a cross branch between the ports, then the port condition will be destroyed, which means, the new four-terminal network is no longer the two port network.

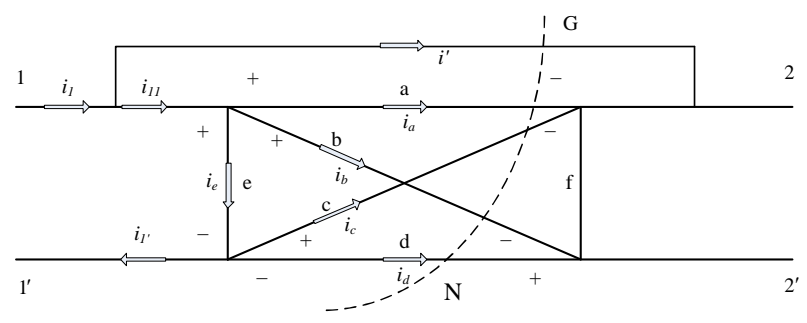

Fig. 4. Two-port conditions failure

The original two-port network meets the current constraint of (5), so for the new network which adding a cross branch, the current algebra to the Gauss surface $G$ is shown in (6). Obviously, the result is not identically zero, so the fourterminal network is not a two-port network.

$$
i_{a}+i_{b}+i_{c}+i_{d}+i^{\prime} \neq 0
$$

\section{INTERCONNECTION OF TWO-PORT NETWORKS}

\section{A. Parallel Connection of Two-Port Networks}

Fig. 5 represents the four terminal network ' $N$ ' formed by two port network ' $\mathrm{N}_{1}$ ' and ' $\mathrm{N}_{2}$ ' in parallel', its NIF topology can be composed of two ports ' $\mathrm{N}_{1}$ ' and ' $\mathrm{N}_{2}$ ' corresponding branches parallel. That is, the non associated branch admittance of the four terminal network ' $\mathrm{N}$ ' is equal to the corresponding branch admittance of the two port ' $\mathrm{N}_{1}$ ' and ' $\mathrm{N}_{2}$ '. It is possible to split the new four terminal network again, so that the port voltage of the two two ports ' $\mathrm{N}_{1}$ ' and ' $\mathrm{N}_{2}$ ' after split is always equal to the port voltage $U_{1}$ and $U_{2}$ in parallel, as shown in Fig. 6 . Then the voltage of the corresponding branches of the two two ports ' $\mathrm{N}_{1}$ ' and ' $\mathrm{N}_{2}$ ' is the same. The condition of the two port derived from the previous derivation obtain (7). In the simultaneous of (7), (8) are obtained. It can be seen that as the Gauss surface $G$, which intersects the non associated branch of the four terminal network ' $N$ ', currents flow into $\mathrm{G}$ remain zero. It is proved that the four-terminal network formed by the two two-ports is also a two-port network.
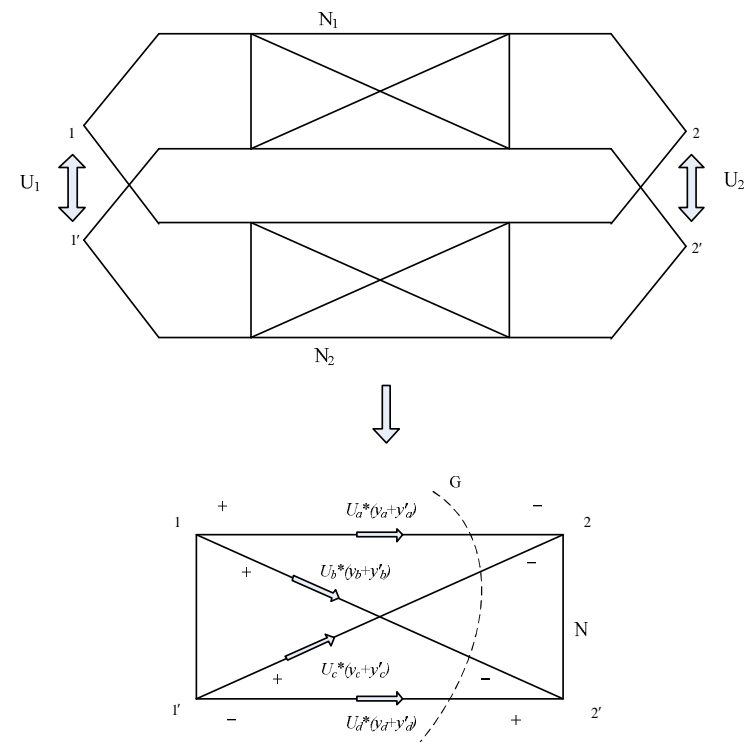

Fig. 5. Parallel of Two-port networks
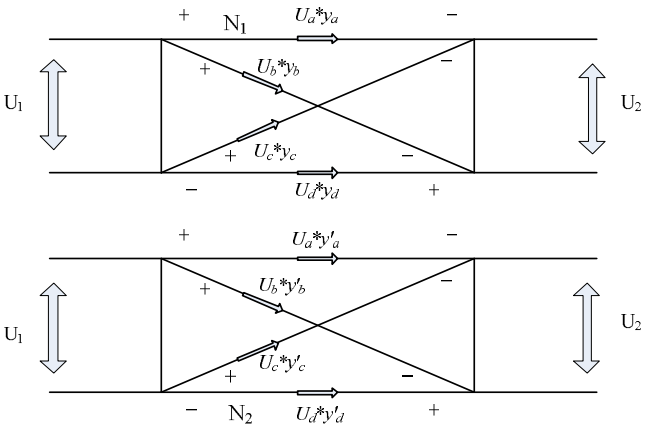

Fig. 6. Separation of paralleled Two-port network 


$$
\begin{gathered}
U_{a} * y_{a}+U_{b} * y_{b}+U_{c} * y_{c}+U_{d} * y_{d}=0 \\
U_{a} * y_{a}^{\prime}+U_{b} * y_{b}^{\prime}+U_{c} * y_{c}^{\prime}+U_{d} *^{\prime} y_{d}=0 \\
U_{a} *\left(\mathrm{y}_{a}+y^{\prime}{ }_{a}\right)+U_{b} *\left(\mathrm{y}_{b}+y_{b}^{\prime}\right) \\
+U_{c} *\left(\mathrm{y}_{\mathrm{c}}+y_{c}^{\prime}\right)+U_{d} *\left(\mathrm{y}_{d}+y_{d}^{\prime}\right)=0
\end{gathered}
$$

\section{B. Series Connection of Two-Port Network}

A four-terminal network ' $\mathrm{N}$ ' formed by the two-port networks is represented in Fig. 7. Split ' $N$ ' from the common nodes ' 0 ' and ' 0 ', as shown in Fig. 8. Suppose that the constant of $U_{1}$ and $U_{2}$ is equal to $U$, and that the unity of $U^{\prime}{ }_{1}$ and $U^{\prime}{ }_{2}$ is equal to $U^{\prime}$, then (9) can be obtained according to the previous derivation. Back to Fig. 7, for the public node ' 0 ', according to the KCL, currents flowing in is equal to the current flowing out of the node. Thus, (10) can be obtained. Through simultaneous (9) and (10), (11) will be obtained. It can be a result that four-terminal network ' $N$ ' which series connected by the port-two ' $\mathrm{N}_{1}$ ' and ' $\mathrm{N}_{2}$ ' is a two-port.

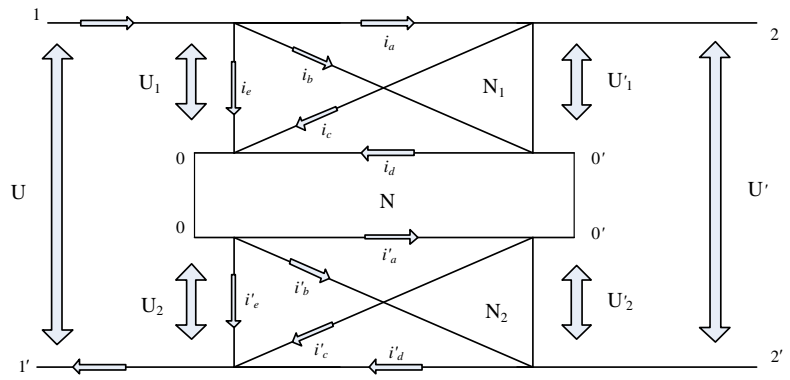

Fig. 7. Series of Two-port networks

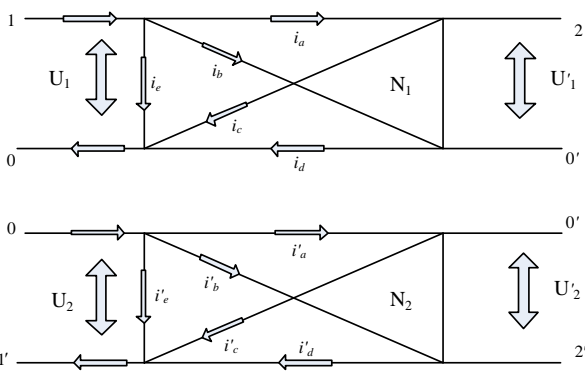

Fig. 8. Separation of seried Two-port network

$$
\begin{gathered}
i_{a}+i_{b}+i_{e}=i_{c}+i_{d}+i_{e} \\
i_{a}^{\prime}+i_{b}^{\prime}+i_{e}^{\prime}=i_{c}^{\prime}+i_{d}^{\prime}+i_{e}^{\prime} \\
i_{c}+i_{d}+i_{e}=i_{a}^{\prime}+i_{b}^{\prime}+i_{e}^{\prime} \\
i_{a}+i_{b}+i_{e}=i_{c}^{\prime}+i_{d}^{\prime}+i_{e}^{\prime}
\end{gathered}
$$

\section{CONDITIONS FOR PARALLEL CONNECTION OF TWO-PORTS}

The judgment condition of two port effective parallel connection has been disgusted in reference [11-15]. In Fig. 9, the condition that the two-port network ' $\mathrm{N} 1$ ' and ' $\mathrm{N} 2$ ' are effectively parallel is that both $\mathrm{U}_{\mathrm{p}}$ and $\mathrm{U}_{\mathrm{q}}$ are zero for any voltage input $\mathrm{U}_{1}$ and $\mathrm{U}_{2}$.
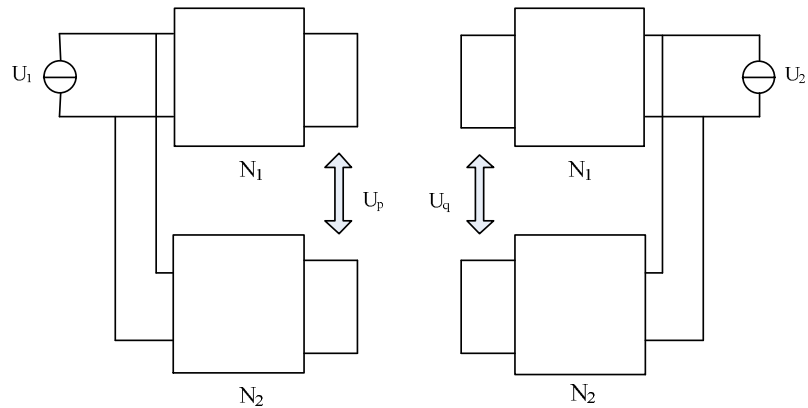

Fig. 9. Judgment of valid paralleled of two-port networks

Analyzing the effective parallel condition of two-ports by NIF topology, and the Fig. 9 can be changed into two-port NIF topology representation, as shown in Fig. 10. Obviously, only by satisfying (12) can make $U_{p}$ constant to zero. Similarly, for the right case in Fig. 9, when the (13) is satisfied, $\mathrm{U}_{\mathrm{q}}$ constant to zero. Therefore, only (12) and (13) are meet that the parallel could be effective.
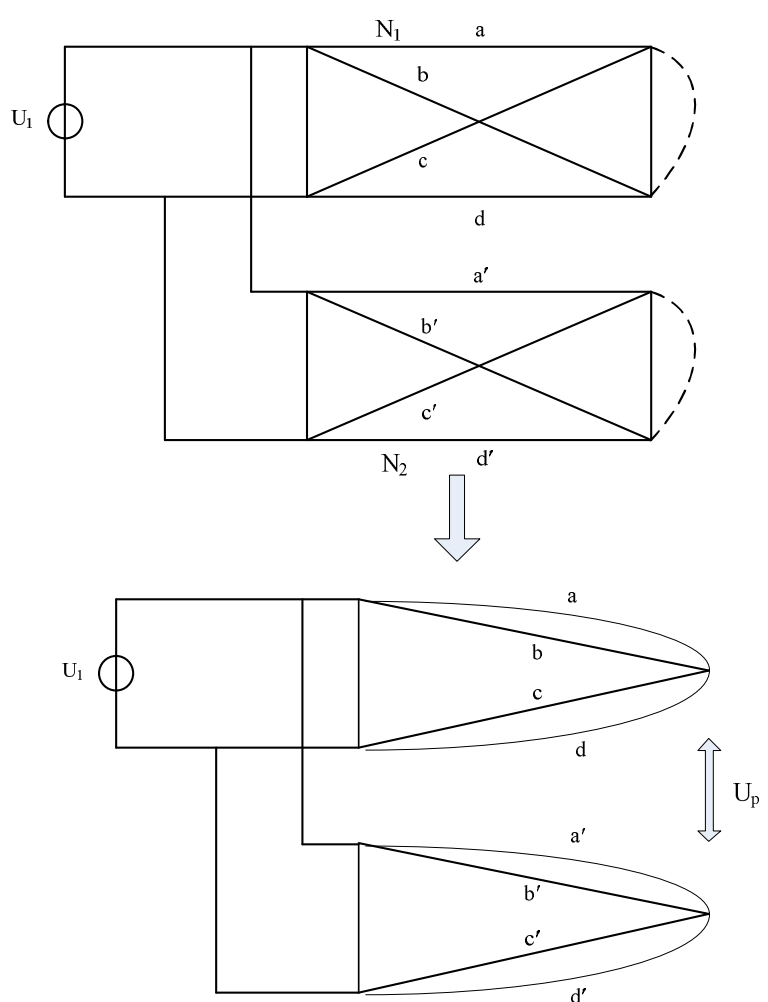

Fig. 10. Judgment of valid paralleled of two-port networks with NIF topology 
An example is given to illustrate the effectiveness of two port parallel decision. Fig. 11 shows three simple two ports ' $\mathrm{N}_{1}$ ', ' $\mathrm{N}_{2}$ ' and ' $\mathrm{N}_{3}$ ', and their NIF topologies can be represented as shown in Fig. 12. In the literature [8], the calculation method of each branch parameter of NIF topology is described in detail. Table 1 gives the admittance of each branch of ' $\mathrm{N}_{1}$ ', ' $\mathrm{N}_{2}$ ' and ' $\mathrm{N}_{3}$ ' NIF topology respectively.
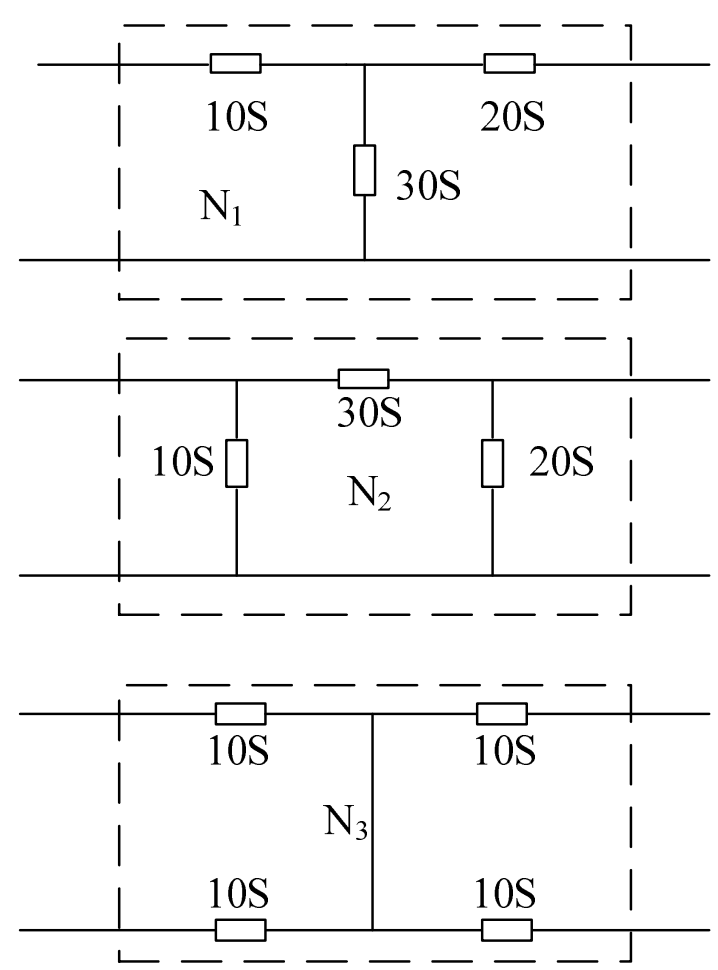

Fig. 11. Simple two-port networks

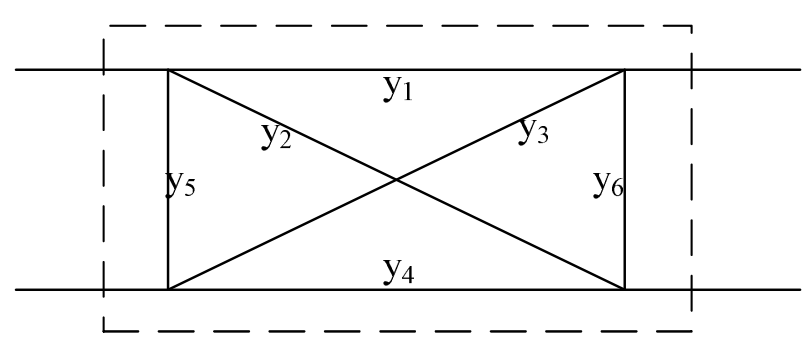

Fig. 12. NIF topology of two-port networks

TABLE 1. PARAMETERS OF SIMPLE TWO-PORT NETWORKS

\begin{tabular}{cccc}
\hline $\begin{array}{c}\text { Admittance }(\mathrm{S}) \\
\text { Itwo-port }\end{array}$ & $\mathrm{N}_{1}$ & $\mathrm{~N}_{2}$ & $\mathrm{~N}_{3}$ \\
\hline $\mathrm{y}_{1}$ & $10 / 3$ & 30 & $5 / 2$ \\
$\mathrm{y}_{2}$ & $25 / 3$ & 40 & 5 \\
$\mathrm{y}_{3}$ & $-5 / 6$ & -25 & 0 \\
$\mathrm{y}_{4}$ & $-65 / 6$ & -45 & $5 / 2$ \\
$\mathrm{y}_{5}$ & $-10 / 3$ & -30 & 15 \\
$\mathrm{y}_{6}$ & $65 / 6$ & 45 & 5 \\
\hline
\end{tabular}

$$
\begin{aligned}
& \frac{y_{a}+y_{b}}{y_{c}+y_{d}}=\frac{y_{a^{\prime}}+y_{b^{\prime}}}{y_{c^{\prime}}+y_{d^{\prime}}} \\
& \frac{y_{a}+y_{c}}{y_{b}+y_{d}}=\frac{y_{a^{\prime}}+y_{c^{\prime}}}{y_{b^{\prime}}+y_{d^{\prime}}}
\end{aligned}
$$

Assuming that ' $\mathrm{N}_{1}$ ' and ' $\mathrm{N}_{2}$ ' are parallel, according to the (12), (13), (14) and (15), it can be concluded that the parallel connection of the two ports ' $\mathrm{N}_{1}$ ' and ' $\mathrm{N}_{2}$ ' is effective. For the ' $\mathrm{N}_{3}$ ', it can go to (16), and it means that ' $\mathrm{N}_{3}$ ' is invalid in parallel whether with ' $\mathrm{N}_{1}$ ' or ' $\mathrm{N}_{2}$ '.

$$
\begin{gathered}
\frac{y^{N_{1}}+y_{2}^{N_{1}}}{y^{N_{1}}{ }_{3}+y^{N_{1}}{ }_{4}}=\frac{y^{N_{2}}+y^{N_{2}}}{y^{N_{2}}+y^{N_{2}}}=-1 \\
\frac{y^{N_{1}}+y^{N_{1}}{ }_{3}}{y_{2}^{N_{1}}+y^{N_{1}}{ }_{4}}=\frac{y^{N_{2}}+y^{N_{2}}}{y^{N_{2}}{ }_{2}+y^{N_{2}}}=-1 \\
\frac{y^{N_{3}}+y^{N_{3}}}{y^{N_{3}}+y^{N_{3}}}=3 \\
\frac{y^{N_{3}}+y^{N_{3}}}{y^{N_{3}}+y^{N_{3}}}=\frac{1}{3}
\end{gathered}
$$

\section{CONCLUSION}

In this paper, the NIF equivalent topology of multi terminal network is applied to the analysis of two port network, and the condition of two ports are found. It is proved that the fourterminal network with two-ports in series and parallel must be a two-port network, so the two port theory is supplemented. On the other hand, a new judgment is proposed for the effective parallel connection of two ports. This method is more practical in the frequency domain analysis of two ports, especially in the wide band analysis of complex two ports.

\section{REFERENCES}

[1] Network T. Two-port Network. Betascript Publishing, 2013.

[2] D.M. Warren, "Automated parameter fitting of two-port network transducer models". J. Acoust. Soc. Am. vol. 135, no. 4, pp. 2339-2339, 2014.

[3] Z.Y. Zhang and G.S. Liang. "A High Frequency Two-port Circuit Model for Potential Transformer Based on the Scattering Parameter". Proc. Chinese Soc. Electr. Eng. vol. 27, no. 27, pp. 39-43, 2007.

[4] S.S. Wang, S.J. Xie, Y. Jin. "Building of 2-Port Network for Ferrite Core Choke Considering the Effects of Parasitic Parameters". Trans. China Electrotech. Soc. vol. 5, pp. 1-8, 2010.

[5] Y. Zhao, J.L. He. "Analysis of Lightning Surge Impedance and Twoport Circuit Model of Transmission Tower", High Volt. Eng. vol. 40, no. 9, pp. 2911-2916, 2014.

[6] L.L. Shi, C.C. Xu. "Theory of Scatter Parameters of Two Port Network and Its Application in the Test of Planar EMI Filter", Trans. China Electrotech. Soc. vol. 28, no. 2, pp. 78-85, 2013. 
[7] Y.Z. Zhu and D.N. Ni. "Design of Quasi-Elliptic Filter with SourceLoad Cross-Coupling", J. Electr. Inform. Tech. vol. 30, no. 3, pp. 604606, 2008.

[8] Y. Liu, S.A. Sebo, R. Caldecott, et al. "Modeling of converter transformers using frequency domain terminal impedance measurements". IEEE Trans. Power Deliv. vol. 8, no. 1, pp. 66-72, 1993.

[9] R. Caldecott, Y. Liu, S.A. Sebo, et al. "Measurement of the frequency dependent impedance of major station equipment". IEEE Trans. Power Deliv. vol. 5, no. 1, pp. 474-480, 1990.

[10] Y. Liu, S.A. Sebo, R. Caldecott, et al. "Power transformer resonancemeasurements and prediction”. IEEE Trans. Power Deliv. vol. 7, no. 1, pp. 245-253, 1992.

[11] D.P. Tian, F. Zhang. "Criterion and Realization of Effective Interconnection of Two-Port Networks", J. Electr. Electron. Edu. vol. 27, no. 5, pp. 31-33, 2005.

[12] C. Bachiller, H.E. Gonzalez, V.E.B. Esbert, et al. "Efficient Technique for the Cascade Connection of Multiple Two-Port Scattering Matrices". IEEE Trans. Microw. Theor. Tech. vol. 55, no. 9, pp. 1880-1886, 2007.

[13] G.K. Behler and M. Makarski, "Two-Port Representation of the Connection between Horn Driver and Horn”. J. Audio Eng. Soc. vol. 51, no. 10, pp. 883-897, 2003.

[14] G. Yao. "The Inverse Transmission Papameters of Series, Parallel, Cascade Connection in the Two-Port Networks". J. Xuzhou, Normal University, 1994.

[15] G. Yao. "The Parameters of Series, Parallel, Cascade Connection in the Two-Port Networks". J. Xuzhou, Normal University, 1993. 